\title{
Un Alfabeto Fonético Internacional en Braille para el Nivel Superior en Argentina
}

An International Phonetic Alphabet in Braille in Argentinean Higher Education

\author{
Carolina Mahler \\ carolinamahler@gmail.com \\ Universidad Nacional de Córdoba, Argentina
}

Recepción: 03 Julio 2019

Aprobación: 08 Julio 2020

Publicación: 01 septiembre 2020

Cita sugerida: Mahler, M. (2020). Un Alfabeto Fonético Internacional en Braille para el Nivel Superior en Argentina. Archivos de Ciencias de la Educación, 14(17), e080. https://doi.org/10.24215/23468866e080

\begin{abstract}
Resumen: Para transformar la barrera del Alfabeto Fonético Internacional (AFI) en la modalidad visual, este trabajo propone su adecuación a Braille. Se propone AFI como accesible y específico para el estudiantado con discapacidad visual en fonética del inglés como espacio curricular en carreras de nivel superior $\mathrm{y}$ universitario argentino, respondiendo tres cuestiones: que AFI es indispensable para la enseñanza y el aprendizaje de la pronunciación del inglés en su especificidad; que la enseñanza de la pronunciación mediante la modalidad oral sola es insuficiente y desiguala el acceso al conocimiento; y, finalmente, que el alumnado es hispanoparlante. AFI transcripto a Braille promueve el aprendizaje multimodal de la pronunciación del inglés, como también autonomía para el trabajo académico y profesional cotidiano, dentro de un marco legal y ético que remite al modelo social de la discapacidad.
\end{abstract}

Palabras clave: Pronunciación del inglés, Braille, Accesibilidad, Multimodalidad.

Abstract: In order to transform the barrier of the International Phonetic Alphabet (IPA) in its visual mode, this article proposes an adaptation to Braille. An accessible specific IPA is proposed specifically for visually disabled students of the English phonetics course in Argentinean higher education programs, while addressing three questions: that IPA is essential for teaching and learning English pronunciation specifically; that teaching pronunciation via the oral mode alone is insufficient and hinders equality of access to knowledge; and that these students are native speakers of Spanish. IPA transcribed into Braille promotes multimodal learning of the pronunciation of English as well as autonomy in daily academic and professional practice, within the legal and ethical framework that refers to the social model of disability.

Keywords: English pronunciation, Braille, Accessibility, Multimodality. 


\section{INTRODUCCIÓN}

En Argentina, cada vez más casas de estudio del nivel superior y universitario articulan acciones vinculadas al acceso a la educación como un derecho de todos. Como en otros 50 países, los principios de la Convención Internacional sobre los Derechos de las Personas con Discapacidad y su Protocolo Facultativo (ONU, 2006) gozan de adhesión supraconstitucional en esta nación. En nuestro caso, la ley 26.378 (2008) versa acerca de los derechos de las personas con discapacidad. A la luz de estos principios y de la aplicación de la ley mencionada en el ámbito de la educación superior, se amparan los derechos a la educación superior de las personas con discapacidad. Además, la Ley de Educación Superior $n^{\circ} 25.573$ incorpora la accesibilidad como responsabilidad de las instituciones de educación superior en su modificatoria 25.573/02. Posteriormente, l a modificatoria 27.204/15, caracteriza como parte de la educación superior tanto la formación de las i nstituciones terciarias como universitarias.

Sin embargo, todavía resta trasladar los mencionados principios y la normativa local a la práctica educativa concreta en todos los espacios. Materializar estos principios implicaría, ante todo, volver a pensar sobre las cuestiones específicas que, por acción u omisión, se han constituido históricamente como barreras de acceso al conocimiento. Esto es precisamente lo que este trabajo pretende repensar en el área de lenguas extranjeras, en particular en la enseñanza de la fonética del idioma inglés.

La noción de barrera pertenece al modelo social de la discapacidad, cuya orientación está interpretada y compilada muy claramente en Barton (2008) y Palacios (2008). Este modelo intenta superar las modalidades estereotípicas históricas de percepción de la discapacidad como enfermedad que requiere una rehabilitación. Además, pretende superar aquellas formas de pensamiento que han asumido como normal que quienes tienen una discapacidad tengan, además, una sobrecarga exclusiva a los fines de lograr una adaptación al entorno físico y a las formas de vida no pensadas desde la diversidad. En el modelo social, identificar las barreras de acceso al conocimiento es solo el primer paso para que posteriormente se logre un acceso equitativo a la educación como un derecho, tal como se promueve en los mencionados principios y normas en Argentina. Sin menoscabo de las discapacidades puntuales de las personas en cuestión, variadas en tipología y grados, el modelo social de la discapacidad permite detectar, sacar a la luz e intentar derribar aquello que el entorno presenta como obstáculo, en lugar de como garantía, de acceso al conocimiento. En particular, y a los efectos de la discusión en este trabajo, adherir a este modelo social permite pensar a las personas con una discapacidad visual como personas que tienen la plena capacidad de aprender la pronunciación del inglés en carreras del nivel superior y universitario. A continuación, se reflexionará sobre un recurso propio de la enseñanza de la pronunciación del inglés en varias carreras del nivel superior en Argentina, específicamente en tanto tal recurso es utilizado en el formato visual. La modalidad visual, por supuesto, resulta inadecuada para las personas con discapacidad visual, es decir, constituye una barrera para la educación formal en la lengua extranjera inglés. Por un lado, el presente artículo revela esta inadecuación. Por otro, ofrece una propuesta orientada a resolverla.

A lo largo del estudio, subyacen las preguntas: ¿cómo se hacen disponibles los recursos que, para cierto contenido curricular, son esenciales? y ¿cómo se hacen accesibles dichos recursos para la totalidad del alumnado? Estas preguntas se asumen como fundamentales. A partir de estos interrogantes, se pretende avanzar reflexivamente en torno de razones a favor de una adecuación de los recursos, transformándolos a accesibles en función de las problemáticas reales en el espacio curricular de la fonética y fonología del idioma inglés en un contexto educativo de habla hispana como primera lengua.

Este trabajo ofrece, más particularmente, una defensa del argumento a favor de la adaptación sistemática y específica, para estudiantes hispanoparlantes con ceguera o baja visión, de los fonemas del idioma inglés en la modalidad táctil (háptica), es decir, al sistema Braille, para la enseñanza y el aprendizaje de la fonética inglesa como espacio curricular en el nivel superior y de grado. Esta propuesta intenta atender la necesidad de adecuar un recurso necesario para el desarrollo óptimo del aprendizaje de la materia. Para este contexto 
y este alumnado, el recurso se torna potencialmente más robusto en impacto y economía cognitivos que el solo uso de la modalidad oral para la formación en el área de fonética del inglés. Como se ha mencionado, en lugar de que tal recurso persista como barrera, en la modalidad visual, para el estudiantado con ceguera o baja visión, su adecuación a Braille lo transforma en accesible y equivalente a los materiales y recursos que tiene el alumnado sin ninguna disminución visual.

La accesibilidad de los fonemas en Braille no quedaría replegada al formato papel de modo exclusivo. En la actualidad existen tablets y libros electrónicos con formato de lectura en Braille, mediante los cuales el estudiantado podría utilizar la modalidad táctil para leer apuntes y libros en la universidad. Este avance tecnológico hace posible la preparación de materiales educativos publicables y compartibles en la accesibilidad, tales como manuales de pronunciación del inglés codificados íntegramente en Braille. De este modo, el estudiantado hispanoparlante con ceguera o baja visión podría elegir, dadas óptimas condiciones, entre el formato legible como lectura en voz alta (voiceover), el formato papel o incluso el digital táctil.

\section{LA PRONUNCIACIÓN DEL INGLÉS COMO ESPACIO CURRICULAR EN CARRERAS DEL NIVEL SUPERIOR Y UNIVERSITARIO}

Una gran cantidad de intervenciones y de proyectos institucionales se han suscitado a lo largo de los años en pos de la integración de personas con alguna discapacidad en los niveles inicial, primario y secundario en Argentina, aunque no sin obstáculos o problemas (Cobeñas, 2015). Sin embargo, la presencia de estudiantado con baja visión o ceguera en el nivel superior o universitario, aunque escasa, es resultado de los logros en estos niveles anteriores, en probablemente casi todas las dimensiones de la vida y en las instituciones que rodean al alumnado.

Cuando las personas con ceguera o baja visión eligen las carreras de nivel superior o de grado como profesorados y traductorados en inglés, licenciaturas de inglés y de dirección de coro, o la carrera de locución, se suman a trayectos de formación prestigiosos como profesionales del idioma inglés en los primeros tres casos, y de la voz cantada y hablada, o de la comunicación en múltiples formatos mediales, en los últimos dos casos. En los profesorados, traductorados y licenciaturas de inglés, la materia pronunciación del inglés, o fonética y fonología del inglés, suele integrarse sucesivamente en varios semestres o años de la carrera, y puede aparecer bajo diferentes denominaciones aunque el contenido sea predominantemente la pronunciación de este idioma. En general, el ejercicio profesional en torno de estas carreras orientadas al inglés es en la docencia del idioma, la traducción pública o la investigación. Diferentes universidades abordan distintas estrategias en torno al ingreso a estas carreras, por ejemplo mediante cursos introductorios para promover el acceso y permanencia en la universidad, o mediante ciclos de nivelación, en algunos casos con la expectativa de logro explícita de habilidades lingüísticas del "nivel umbral" (Consejo de Europa, 2002). Durante la carrera, los espacios curriculares relacionados con la pronunciación del inglés exigen dar cuenta de aspectos teóricoprácticos fonemáticos, prosódicos y sociodialectales, de dominio de la lengua frente a cualquier situación comunicativa y profesional. En otras carreras, como en las licenciaturas en dirección coral o en locución, la pronunciación del inglés se reduce generalmente un solo seminario o curso semestral. En carreras como locución o dirección de coro, no se recomienda ninguna competencia lingüística particular en inglés en el ingreso. Por ello, el estudiantado suele ser multinivel, de acuerdo con la orientación en su trayecto previo en la escuela media, en academias o viajes, y actividad en redes sociales, por ejemplo. Al finalizar el trayecto formativo en locución, se logra la habilitación al ejercicio profesional en todo el territorio nacional argentino mediante el título de locutor integral otorgado por la institución donde curse su carrera, y la habilitación regulada por el Instituto Superior de Enseñanza Radiofónica (ISER), perteneciente al Ente Nacional de Comunicaciones (ENACOM). Así, al egresar, cada institución de nivel superior o universidad certifica que el alumnado de la carrera correspondiente se ha formado en habilidades relacionadas con la pronunciación de un idioma (o varios) como lengua extranjera. 
En este marco general en Argentina (así como en muchos otros países), el inglés ocupa un lugar privilegiado como idioma extranjero en estas carreras, por ser lingua franca en el mundo. Se suelen evidenciar ciertas problemáticas idiosincrásicas a partir del hecho de que salvo casos muy escasos y excepcionales, el estudiantado es hispanoparlante. En particular, al respecto de la relación entre la forma escrita y la oralidad (Marks, 2007; Tench, 2011), que se discutirá en torno a la discapacidad visual más adelante, y al respecto de ciertos rasgos enunciativos en la oralidad propios del habla en castellano en Latinoamérica y Argentina (Finch y Ortiz Lira, 1982), cuyos rasgos prosódicos y fonemáticos ingresan, en tanto es primera lengua, desde la variedad dialectal local, al inglés. Este punto excede la problemática puntual del trabajo, por lo que no se realizarán descripciones de dichos rasgos. Basta destacar la importancia de que todo el estudiantado, con o sin ceguera, es hispanoparlante de alguna variedad local. A estas problemáticas, se suman las particularidades del estudiantado con ceguera o baja visión (HCBV de ahora en más), futuros profesionales del inglés o de la voz en el arte y en los medios de comunicación.

\section{EL ALFABETO FONÉTICO INTERNACIONAL}

El Alfabeto Fonético Internacional (AFI) o International Phonetic Alphabet (2007) (IPA, en su habitual acrónimo en inglés) es un recurso esencial para la enseñanza y aprendizaje de la pronunciación del inglés (y de otras lenguas). Es la representación sistemática y simbólica del inventario de todos los sonidos posibles e ideales que, además, se especifica para lenguas naturales particulares. Como sistema de notación escrita de la oralidad pura, alivia la complejidad ortográfica y las irregularidades y superposiciones de distintas letras del alfabeto para un mismo sonido. Por ejemplo, para AFI en español (Real Academia Española y Asociación de Academias de la Lengua Española, 2011), el primer sonido en las palabras casa, queso y kiosco se representaría en un solo fonema: / $\mathrm{k} /$. Esto se debe a que en AFI se codifica un mismo sonido como un solo símbolo fonético escrito, y así se evitan las duplicaciones o superposiciones, habituales en la ortografía de muchos idiomas. El principio de parsimonia subyace a la transcripción de la oralidad.

AFI cumple muchas funciones, además de representar sonidos (fonemas) para la enseñanza de idiomas extranjeros. Históricamente ha servido para salvaguardar el registro anotado escrito de lenguas puramente orales, como es el caso de muchas lenguas de América, lo que permite su estudio y preservación, en particular de aquellas lenguas que desafortunadamente tienen cada vez menos hablantes nativos. También puede representar las diferentes variedades que se ajustan a las normas y convenciones de grupos dentro de una misma lengua. Además, puede ser utilizado por especialistas de la voz, para registrar impedimentos o dificultades en el habla de pacientes (por ejemplo, en foniatría), para detectar problemas de dicción y mejorar la interpretación en el canto, y mucho más.

En su codificación de fonemas, AFI utiliza gran parte de las letras que ya son conocidas en la comunidad de habla argentina: el alfabeto latino. Por ello, la curva de aprendizaje de AFI, en un curso de pronunciación del inglés en un país como Argentina, es mucho menor que si el estudiantado desconociera dicho alfabeto. Así y todo, AFI contiene algunos diacríticos y letras del alfabeto griego, y requiere de instrucción, tiempo y aplicación. Sin embargo, su función primaria es facilitar la distinción y precisión articulatoria de los sonidos provenientes del repertorio de, en el caso que nos ocupa, el inglés.

Quien desconozca la utilidad de AFI para aprender inglés se preguntará por qué no utilizar el alfabeto que utilizamos en nuestra lengua escrita, sin más. De modo crucial, este último no distingue múltiples fonemas en inglés. Por ejemplo, no logra representar la diferencia enunciativa (oral) de las siguientes palabras: cat (gato) - cut (cortar) - cart (carrito), para un hispanoparlante. Siguiendo el ejemplo, AFI especifica, mediante la

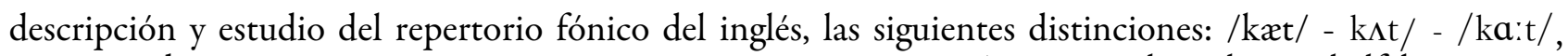
correspondientemente. Entonces, una persona sin instrucción en AFI, que solo utilizara el alfabeto propio de la escritura del español, intentaría distinguir semántica y fonológicamente entre estas tres palabras del inglés, pero solo podría representarlas como *kat, o peor aún *cat, confundiendo las tres pronunciaciones 
distintas como una sola, o subsumiendo tres diferentes significados en uno solo, i.e. ${ }^{*}$ gato (el asterisco indica error). Este es un grave problema. Por no distinguir estos fonemas vocálicos, o cualesquiera otros fonemas en otros casos, se perjudica la distinción semántica para sí y para su recepción por el auditorio, y por lo tanto pone en jaque el contenido de lo enunciado en términos comunicativos. Entorpecer o imposibilitar la comunicación es inadmisible para cualquier profesional en la enseñanza, traducción o investigación del inglés, así como para artistas que intenten comunicar algo cantando, como también para quienes trabajen en los medios como comunicadores, dado que la comunicación es el mismísimo material que compone la trama que les ocupa. Así, AFI es un recurso indispensable para la enseñanza y aprendizaje de la pronunciación de idiomas extranjeros en general, y del inglés en particular.

\section{PARTicularidades EN LA ClASE DE FONÉTICA INGLESA: AFI COMO BARRERA EN EL FORMATO VISUAL EXCLUSIVAMENTE}

En el contexto real de los cursos de pronunciación del inglés en el nivel superior en Argentina, el material con el que cuenta el estudiantado incluye típicamente audios, podcasts, enlaces web (modalidad auditiva y audiovisual) y libros de texto (modalidad visual). En este material se representan los sonidos meta (audio) y los fonemas en su formato escrito en AFI (visual), para el entrenamiento en este alfabeto fonético y el desarrollo de las capacidades de recepción y articulación del repertorio fónico del inglés.

En lo que refiere a las modalidades sensoriales, quienes no tienen ceguera o baja visión cuentan, además del entrenamiento en la percepción acústica, con el recurso visual de representación gráfica de cada fonema en el repertorio del inglés. De ese modo, tienen también un registro visual, pueden tomar notas y volver a ellas o al material bibliográfico cuantas veces lo requieran. Las impresiones acústicas y los signos escritos que AFI representa quedan vinculados entre sí.

En cambio, para el estudiantado con ceguera o baja visión, la percepción acústica de los fonemas y la práctica oral suele ser, como regla general, la única modalidad dentro de la cual la pronunciación se modela y practica en los cursos específicos de fonética y fonología inglesa -e incluso de inglés general. Esto significa que, a nivel perceptivo, no existe un correlato que acompañe el registro perceptivo de lo acústico en ninguna otra modalidad. Además, el alumnado HCBV no cuenta con ningún libro o manual en formato legible como lector de texto de AFI a audio, debido a que los programas de accesibilidad más utilizados no pueden "leerlos". El hecho es que muchos de los símbolos fonéticos no pertenecen al alfabeto ni del castellano ni del inglés, y el lector a audio no los reconoce. Así pues, el recurso de AFI se torna inutilizable en estas condiciones. No obstante, AFI sigue siendo indispensable para el aprendizaje y la enseñanza de la pronunciación del inglés.

\section{LA PRopuesta: Braille EN IPA ESPEcífico}

La propuesta persigue la idea de que el material de cátedra de fonética del inglés en formato escrito sea transcripto a Braille de modo completo, de modo tal que las personas con o sin ceguera y baja visión, en una clase, puedan acceder al recurso de AFI para el desarrollo de los contenidos específicos de pronunciación del inglés. La perspectiva de este trabajo asume que las personas HCBV deberían acceder a los mismos o equivalentes recursos y materiales que aquellas que no tienen $\mathrm{CBV}$-como mínimo. A su vez, la propuesta contempla las opciones actuales del Braille, en formatos de e-book y tablet con versión Braille integradas, además de la versión más tradicional en papel.

La adaptación del formato del material se concibe bajo el criterio de accesibilidad del recurso específico, el cual conservaría las mismas funciones y usos que en su formato de origen. La propuesta atiende tres cuestiones: en primer lugar, que AFI es indispensable para la enseñanza y el aprendizaje de la pronunciación del inglés como asignatura específica. En segundo lugar, la enseñanza de la pronunciación mediante la 
modalidad oral exclusivamente, en estas condiciones y para este contenido específico, es insuficiente y promueve desigualdad de acceso al conocimiento. En tercer lugar, el estudiantado es hispanoparlante, todo lo cual ocasiona la necesidad de una diferenciación del AFI utilizado por angloparlantes CBV, y creación de un AFI para el contexto de nuestras casas de estudio en Argentina.

\section{Problemas Por Resolver: AFI ACCESIBLE COMO SOLUCión}

Una primera problemática es la diferencia insalvable, entre el castellano y el inglés, al respecto de la correspondencia entre fonemas y letras. La escritura como parte de la cultura, en inglés, es que "no es una escritura 'fonmica', entendida como una correspondencia estricta entre fonemas y letras" (Halliday y Matthiessen, 2004, p. 19) (la traduccin es ma). En inglés, nunca se lee como se escribe: simplemente se lee como se pronuncia. Por ejemplo, no es extraño que una letra vocal pueda tener un valor consonántico (por ejemplo, el primer fonema en la palabra university es la semiconsonante / $\mathrm{j} /$ ); o que un dígrafo como "th" se pronuncie como un solo fonema - pero cuál fonema es impredecible a partir de las letras "th". En esta lengua, no es posible recuperar el valor fonémico de "th" solo - debemos conocer cómo se pronuncia la palabra entera. Si no la conocemos, como sucede en los contextos de aprendizaje del inglés como lengua extranjera, AFI vuelve a ser una solución, proveyéndonos de la distinción entre el dígrafo "th", e.g. en this /ðIs/, y en thanks / $\theta$ ænks/, donde puede evidenciarse la diferencia fonética entre dos palabras que contienen "th". Aun más, abundan las excepciones, como en el nombre propio "Thomas", donde "th" se pronuncia /t/. Es claro que algunas pronunciaciones de palabras comunes podrían conocerse por tratarse de vocabulario ya aprendido; s in embargo en los casos de todas las profesiones mencionadas, es importante poder rastrear, identificar y producir las pronunciaciones de vocabulario nuevo también.

Con el español como punto de partida del estudiantado en Argentina, la tendencia es leer textos a partir de la letra escrita, porque así tales estudiantes lo han hecho toda la vida, y porque ello les ha asegurado una adecuada pronunciación de textos -en español. En esta lengua, basta con conocer las reglas de pronunciación a partir de la forma escrita. Un ejemplo de tales reglas en castellano para la letra "g" es que si está seguida de "ue", tal "u" será pronunciada o muda en virtud de llevar diéresis o no, como se puede observar en "gera" y "guerra". Sin embargo, esta propiedad del castellano no tiene un correlato en inglés. Existe una escasa o nula dependencia de reglas de enunciación oral a partir de la forma escrita (Tench, 2011) en el idioma inglés. Por ende, en esta lengua, nunca se puede predecir con certeza la pronunciación de una palabra por su forma escrita. Los ejemplos son innumerables y constitutivos del sistema; las reglas, escasas y con cuantiosas excepciones.

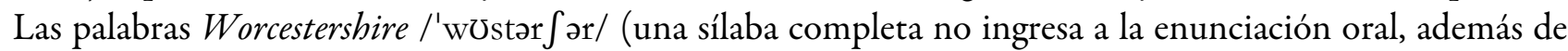
varias letras) y Yosemite /ja'semItI/ son un llamativo ejemplo de impredictibilidad a partir de la forma escrita.

El repertorio fónico del inglés contiene mayor cantidad de vocales que letras del castellano (12 vocales en inglés), y también contiene más consonantes. Por ello, la utilización de letras del alfabeto escrito es insuficiente para la cantidad de fonemas existentes en inglés. De hecho, la representación escrita de la pronunciación del inglés, exclusivamente en letras del alfabeto comn, puede ser un grave obstáculo para la correcta pronunciación del inglés, como se ha explicado en la sección 3. AFI permite aprender todos los sonidos específicos del habla inglesa, reconocerlos como tales, y poner en marcha su enunciación en voz alta. Efectivamente, también posibilita leerlos a partir de un texto escrito, o comprender y enunciar la pronunciación de vocabulario nuevo a partir de diccionarios. Por cierto, los diccionarios online y en papel utilizan AFI como modo de representación de la pronunciación. AFI garantiza un recupero de la forma acstica de la palabra o del texto a pesar de cómo se escriba en el alfabeto que utilizamos cotidianamente, cuestión crucial en el caso de inglés, donde la relación palabra-pronunciación (fonemas) se presenta como azarosa, como se ha visto. A continuación, los símbolos fonéticos que representan sonidos sin una correspondencia con ninguna letra de nuestro alfabeto en español: vocálicos $/ \mathrm{I} /, / \mathfrak{x} /, / \mathrm{D} /, / \Lambda /$, /

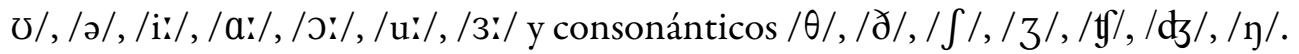


Es importante destacar que para el alumnado hispanoparlante en general -vale decir, tengan o no alguna dificultad visual- la pronunciación del inglés constituye un desafío. Debe perder la confianza y abandonar el registro escrito de las letras (que ya conoce) como soporte para el logro de la enunciación adecuada, particularmente en la pronunciación de vocabulario nuevo. Para el vocabulario conocido, al inicio del curso en pronunciación del inglés, las pronunciaciones suelen ser típicas de la interlengua, articuladas de un modo impreciso o como otras segundas lenguas conocidas, o bien, típicamente, con abundante uso del repertorio fónico de la variedad de español que utiliza cada estudiante. Por todo lo anterior, AFI es imprescindible, e ingresa en la presente propuesta.

Una segunda problemática muy actual en el estudiantado con CBV, habitualmente jóvenes, es la preferencia por la utilización de aplicaciones o software de lectura parlante en computadoras, telefonía celular y audiolibros, entre otras cada vez más apreciadas tecnologías de accesibilidad, por sobre, o en lugar de, la lectura en Braille. Por un lado, esta preferencia es esperable en la actualidad, en particular en la franja etaria que compone el nivel de pregrado y grado; y por otro, la utilización de esta tecnología es deseable, en el sentido de que implica un acceso a cuantiosa información multimedial para la lectura de apuntes y libros completos, por ejemplo. Sin embargo, la conectividad discursiva exclusivamente oral puede afectar la distinción entre un fonema y otro en la lengua extranjera, y por lo tanto, puede también perjudicar el aprendizaje sistemático del repertorio fónico del inglés. De hecho, la oralidad, entendida como principio, recurso y fin, puede constituir un verdadero obstáculo en la distinción de los fonemas. Basta tener en cuenta la brevedad de la enunciación normal de cualquier fonema en el tiempo, en cualquier idioma, junto con el hecho objetivo de que al hablar, ligamos un sonido a otro. De hecho, la separación de fonemas en el habla corriente haría incomprensible cualquier frase, en el habla encadenada de cualquier lengua. En particular, en las clases de pronunciación del inglés, hacer descansar todo el trabajo de pronunciación en la oralidad encadenada o en las formas orales puras, de modo exclusivo, perjudica la atención a la precisión articulatoria de cada uno de los fonemas del inglés, su contraste con el repertorio fónico del castellano, su retención en la memoria y su recuperación, para su reconocimiento posterior y producción espontánea o anticipada. Este es un problema típico del entorno que el estudiantado HCBV, hasta ahora, ha venido enfrentando en soledad.

En la dimensión áulica, el trabajo oral exclusivo, a lo largo de todo el desarrollo del contenido de la asignatura, puede debilitar seriamente la enseñanza y el aprendizaje de la pronunciación del inglés como área específica de conocimiento. En particular, una consecuencia indeseada de ello es que este tipo de trabajo despoja de autonomía al alumnado para la práctica fonémica, la conciencia fonológica y el fortalecimiento de los fonemas en la memoria, haciendo más dificultosa su integración a la memoria de largo plazo. El alumnado dependería de la enunciación oral inmediata de su profesora o profesor, o del audio grabado en la inmediatez del contexto para la práctica o el uso profesional (memoria de corto plazo). En cambio, conforme el alumnado vaya trabajando la distinción de fonemas en clase y fuera de ella, con AFI, hay mayores probabilidades, exceptuando impedimentos de otro tipo, de que logre transferir las distinciones fónicas desde la memoria de corto plazo a la de largo plazo (Hulme, Maughan y Brown, 1991), especialmente en los contextos de novedad léxica y semántica. Cuando estos rasgos orales se vinculan con una representación simbólica de otra índole, pueden retenerse mejor en la memoria, practicarse y, finalmente, apropiarse.

Entonces, la propuesta incluye un aporte desde la modalidad háptica, con el fin de conformar estructuras de representación que sean significativas y distinguibles, contribuyendo a su representación en la vida mental del estudiantado HCBV. Las representaciones mentales subjetivas de las señales acústicas son el resultado de la percepción y la experiencia dentro y fuera de clase de cada estudiante, a lo largo del tiempo. Estas representaciones mentales vinculan el fonema (percepción acústica) y su correspondiente formato escrito ( $\sin \mathrm{CBV}$ ) o, como se defiende en este trabajo, su representación en formato táctil, en Braille (con CBV), que es el equivalente más apropiado para estudiantes HCBV.

Conforme la propuesta incluye Braille, tres significativas ventajas emergen: se reduce el esfuerzo cognitivo, en tanto el estudiantado HCBV puede apoyarse en un formato ya conocido, como podemos asumir, en 
el nivel educativo superior. La segunda ventaja (o tal vez, la ausencia de dificultad) es que, el desafío de trabajar con AFI en Braille, para personas HCBV, es análogo al del proceso de quienes no tienen ninguna discapacidad visual. Como se ha explicitado anteriormente, AFI para videntes también requiere de un proceso que demanda empeño y dedicación. La tercera ventaja es que la utilización de Braille activa la corteza visual en ausencia de visión (Sadato, Pascual-Leone, Grafman, VIbañez, Deiber, Dold y Hallett, 1996). Así, se activarían las mismas áreas cerebrales, mediante distintos canales sensoriales, tanto en una persona sin ceguera como en una con CBV. De este modo, toda la clase, con CVB o no, tendría su correspondiente correlato perceptivo y neural, haciendo la misma tarea, es decir, trabajando con representaciones en AFI.

La tercera y última problemática refiere a la transcripción estandarizada de AFI (IPA, en inglés) a Braille de Estados Unidos (Braille Authority of North America, 2011). Desafortunadamente, esta transcripción exhibe un sesgo lingüístico, puesto que está configurada para angloparlantes nativos exclusivamente. Esta reconocida aplicación de AFI a inglés estadounidense no constituye un herramienta didáctica viable para el escenario de cursos de pronunciación del inglés como lengua extranjera en el contexto descripto en esta propuesta. En efecto, causaría más confusión que representaciones mentales adecuadas, dando lugar a enunciaciones muy probablemente inapropiadas. A modo de ilustración del problema, de entre muchos otros ejemplos que presenta esta versión de IPA en Braille, es el fonema / $\mathrm{u}$ : / (vocal larga). Este fonema se representa como una doble "o", es decir "oo" en Braille estadounidense. El estudiantado HCBV que lea entonces la palabra "blue", por ejemplo, se encontraría con la representación bloo en Braille. La mencionada transcripción no es, de modo alguno, intuitiva para estudiantes de inglés como lengua extranjera.

Es importante pensar, para todos los casos mencionados, que el alumnado va a encontrarse con vocabulario nuevo, con frases y textos novedosos, literarios, fragmentarios y completos que debería poder afrontar con un recurso que, en lugar de entorpecer la pronunciación, arroje claridad y brinde precisión. También es un hecho que en su entorno local circulan pronunciaciones inadecuadas de nombres comunes, propios o marcas. Por todo lo mencionado, y como contrapartida de la barrera que supone la utilización de AFI en modalidad visual exclusivamente o de la ausencia de AFI, la propuesta es crear una versión accesible, en Braille, del Alfabeto Fonético Internacional, para las personas con ceguera o con baja visión que estudian pronunciación del inglés en el nivel superior y universitario en Argentina.

Esta propuesta abraza el principio de parsimonia de AFI, por el cual un mismo símbolo fonético de AFI encaja con una forma fónica del repertorio del inglés, para su utilización específica y realista en este contexto de enseñanza y aprendizaje. El diseño experimental y adecuación al Braille de los posibles alfabetos para estudiantes HCBV excede este trabajo, que debería articular la participación de docentes especiales o de apoyo, de fonética inglesa, de diseñadores capacitados en accesibilidad de materiales, y de modo fundamental, de personas HCBV.

\section{ConCLUSIÓN}

Un recurso accesible en las aulas reales del nivel superior y universitario en Argentina, para la formación en la pronunciación del inglés, debería estar adaptado específicamente a nuestros estudiantes HCBV. Primero, se debe considerar que el inglés no es lengua nativa en Argentina. Segundo, que el inglés es una pieza importante en la formación profesional de locutores habilitados por el ISER-ENACOM, y de cantantes profesionales, y que es fundamental para profesores, traductores y licenciados en la lengua inglesa, en Argentina. Tercero, se debe poner de relieve que en la enseñanza y el aprendizaje de la pronunciación del inglés en el nivel superior y de grado se deben considerar las posibilidades perceptivas en distintas modalidades del estudiantado, y las mejores formas de presentación de materiales disponibles. Por ello, se deben enfatizar aquellas capacidades perceptivas y cognitivas disponibles que contribuyen a la distinción de los elementos que componen el repertorio fónico del inglés. Así, AFI en Braille adaptado a hablantes nativos del castellano emerge como recurso necesario y adecuado, como una solución a lo que de otro modo 
se presenta como barrera. La propuesta no afirma que este recurso con diseño accesible sea la única solución, ni la garantía del aprendizaje de la pronunciación del inglés. Tampoco se desprende de este trabajo que AFI en Braille sea lo único que el estudiantado HCBV necesita: sólo se han brindado razones para que se cree y se incluya en estos contextos reales mencionados.

El estudiantado requiere la remoción de barreras para el aprendizaje, pero además, un alejamiento de la dependencia epistémica. Las motivaciones que defienden la propuesta descripta están orientadas en dirección del empoderamiento epistémico, en dirección opuesta de los modelos de prescindencia y rehabilitación al respecto de las personas con alguna discapacidad. Asimismo, la propuesta considera, para la concreción del diseño del Alfabeto Fonético Internacional accesible en Braille, la participación de estudiantes CBV como actores esenciales en la optimización del recurso, a la par de educadores en el área. Esta propuesta también ha interpretado un contexto específico, pero en tanto está sustentada por normativa vigente en muchos países cuya lengua oficial o de uso no es el inglés, puede extrapolarse y abarcar contextos pedagógicos de mayor alcance que Argentina solamente, conforme adhieran a los principios de la Convención Internacional sobre los Derechos de las Personas con Discapacidad y Protocolo Facultativo (ONU, 2006).

Algunos corolarios importantes a partir del diseño, creación e implementación de AFI en Braille incluyen que podría constituir una herramienta para la pronunciación del inglés en clases de inglés general en los ámbitos previos tales como el secundario, así como en los cursos de grado y posgrado de inglés que requirieran esa herramienta para alguna unidad temática transversal, y no necesariamente para fonética del inglés como materia específica. Finalmente, esta propuesta ha considerado que el recurso posee un enorme potencial para el estudiantado en el ámbito profesional pleno: que egresen profesionales con ceguera o baja visión, y que hagan uso del recurso propuesto, por ejemplo, como docentes en carrera, dota a AFI en Braille de una dimensión empoderadora mucho más plena aun. Hay allí un horizonte de agentividad, crítica y apertura a, y circulación de, conocimiento de la pronunciación del inglés, cuya significatividad puede ser construida y vivida genuinamente entre profesores y estudiantes de inglés HCBV.

\section{REFERENCIAS}

Barton, L. (2008). (Comp.) Superar las barreras de la discapacidad. Madrid: Ed. Morata.

Cobeñas, P. (2015). Buenas prácticas inclusivas en la educación de personas con discapacidad en la provincia de Buenos Aires y desafios pendientes. Buenos Aires: Asociación por los Derechos Civiles. Recuperado de https://educacio n-inclusiva.com.ar/wp-content/uploads/2015/10/Buenas-practicas-Educacion-Inclusiva-ADC-2015.pdf

Finch, D. y Ortiz Lira, H. (1982). A Course in English Phonetics for Spanish Speakers. Londres: Heinemann Educational Books Ltd.

Halliday, MAK. y Matthiessen, C. (revisión) (2004). Introduction to Functional Grammar (3rd ed.). London: Arnold.

Hulme, C., Maughan, S. y Brown, GDA (1991). Memory for familiar and unfamiliar words: Evidence for a long-term memory contribution to short-term memory span.J Mem Lang, 30(3), 685-70

Marks, J. (2007). English Pronunciation in Use. Elementary. Self-study and classroom use. Cambridge: Cambridge University Press.

Palacios, A. (2008). El modelo social de discapacidad: orígenes, caracterización y plasmación en la Convención Internacional sobre los Derechos de las Personas con Discapacidad. Madrid: Ediciones Cinca. Recuperado de: http s://www.cermi.es/sites/default/files/docs/colecciones/Elmodelosocialdediscapacidad.pdf.

Sadato, N., Pascual-Leone, A., Grafman, J., VIbañez, MP., Deiber, MP., Dold, G., Hallett, M. (1996). Activation of the primary visual cortex by Braille reading in blind subjects. Nature, 380(6574), 526-528.

Tench, P. (2011). Transcribing the Sound of English. A Phonetics Workbook for Words and Discourse. Cambridge: Cambridge University Press. 


\section{Documentos}

Argentina, Ley 25.573 (2002).

Argentina, Ley 26.378 (2008). Convención sobre los derechos de las personas con discapacidad.

Argentina, Ley 27.204/15 (2015). Implementación efectiva de la responsabilidad del estado en el nivel de educación superior.

Braille Authority of North America (2011). Braille Formats Principles of Print-to-Braille Transcription. Recuperado de: http://www.brailleauthority.org/formats/2011 manual-web/

Consejo de Europa (2002). Marco común europeo de referencia para las lenguas: aprendizaje, enseñanza, evaluación. Madrid: Anaya. Recuperado de: https://rm.coe.int/1680459f97

Organización de las Naciones Unidas (2006). Convención Internacional sobre los Derechos de las Personas con Discapacidad y Protocolo Facultativo. Recuperado de http://www.un.org/disabilities/documents/convention/c onvoptprot-s.pdf

Real Academia Española y Asociación de Academias de la Lengua Española (2011). Nueva gramática de la lengua española. Fonética y fonología. Madrid: Espasa.

The International Phonetic Association (2007). Handbook of the International Phonetic Association. A guide to the use of the International Phonetic Alphabet. Cambridge: Cambridge University Press. 